\title{
Serum metabolomic profiling of human gastric cancer and its relationship with the prognosis
}

\author{
Daguang Wang ${ }^{1}$, Wei Li ${ }^{1}$, Qi Zou ${ }^{1}$, Lei Yin², Yechao Du ${ }^{1}$, Jingkai Gu² and Jian Suo ${ }^{1}$ \\ ${ }^{1}$ Department of Gastrointestinal Surgery, First Hospital of Jilin University, Changchun, Jilin 130021, China \\ ${ }^{2}$ Clinical Pharmacology Center, Research Institute of Translational Medicine, First Hospital of Jilin University, Changchun, Jilin \\ 130021, China \\ Correspondence to: Jian Suo, email: suojian0066@126.com \\ Keywords: gastric cancer (GC); metabolomics; serum; biomarkers; prognosis \\ Received: May 09, $2017 \quad$ Accepted: August 26, $2017 \quad$ Published: September 28, 2017 \\ Copyright: Wang et al. This is an open-access article distributed under the terms of the Creative Commons Attribution License 3.0 \\ (CC BY 3.0), which permits unrestricted use, distribution, and reproduction in any medium, provided the original author and source \\ are credited.
}

\section{ABSTRACT}

Objective: This study was aimed to investigate serum metabolites in gastric cancer (GC) patients and their relationships with the prognosis of GC in order to find potential specific serum biomarkers for GC.

Methods: Blood samples of 125 GC patients of unifocal GC at initial stage and 38 healthy people recruited in our hospital from September 2008 to August 2009 were analyzed by using high performance liquid chromatography coupled with electrospray ionization/quadrupole-time-of-flight mass spectrometry (HPLCESI/Q-TOFMS). Multiple statistical methods like principal component analysis (PCA), hierarchical clustering analysis, partial least squares discriminant analysis (PLS-DA), multivariate COX regression analysis, variance analysis and $\mathrm{K}-\mathrm{M}$ survival curve were applied to analyze the raw obtained mass data in order to analyze the independent prognostic factors of GC. The structures of these metabolites were confirmed by comparing the $\mathrm{m} / \mathrm{z}$ ratio and ion mode of with the data published from HMDB (www.hmdb.ca) databases.

Results: By PLS-DA test, 16 serum metabolites in ESI ${ }^{+}$mode of VIP $>1$ in both test group and validation group could definitely distinguish GC patients from healthy peoples $(p<0.05)$. Multivariate COX regression analysis showed TNM staging, 2,4-hexadienoic acid, 4-methylphenyl dodecanoate and glycerol tributanoate were independent prognostic factors of GC $(p<0.05)$. In the $\mathrm{K}-\mathrm{M}$ survival analysis, the survival rate in high level group of the 3 selected serum metabolites together or alone was significant lower than in those in low level group $(p<0.05)$.

Conclusion: Low serum levels of 2,4-hexadienoic acid, 4-methylphenyl dodecanoate and glycerol tributanoate may be important independent prognostic factors of GC.

\section{INTRODUCTION}

Developed from the malignant cells in the stomach inner lining, gastric cancer (GC) has high mortality all over the world, commonly in the Eastern countries, such as in China, Korea and Japan [1-3]. Nowadays, therapies for GC consist of chemotherapy, surgery, radiation and targeted therapy [4-6]. As the underlying molecular mechanism of GC is still unknown and the clinical symptoms of early gastric cancer are usually unobvious, there still lacks of effective therapy for GC.

Endoscopy is the most common diagnostic method for the early GC, but the efficiency was inconsistent among different endoscopists and pathologists [6,7]. Although early definition and management at the beginning stage can decrease $\mathrm{GC}$ incidence, the prognosis of $\mathrm{GC}$ remains poor 
and the overall 5-year survival rate is still less than $40 \%$ [4-6]. The current prognosis indicators include pathology findings of histological type, invasion and metastasis, imaging results of classifications and other clinical characteristics like age and underlying diseases, etc., and further therapy method should be determined based on all these indicators. However, there still lots of limitations for these traditional indicators. Currently, biomarkers like p27, cyclin E, E-cadherin, c-erbB2, cmyc, tumor suppressor gene $\mathrm{p} 53$ etc. have been reported to be effective prognosis factors for GC patients. Furthermore, more and more serum metabolomics have been recommended as prognosis indicators in developed countries due to high specificity and sensitivity, which plays a key role in GC therapy [8, 9].

Lots of biological studies show that metabolites in human fluid samples (such as serum, bile, sputum, aqueous humor, etc.) can be important downstream or endpoint biomarkers for gene mutation mutations due to endogenous substance or xenobiotics and are more specific and sensitive to different disease stages [10-14]. As a result, metabolomics plays an important role in current biological research, especially in underlying carcinogenesis and proliferation mechanism and thereby establishing useful biomedical indicators for early diagnosis and management in current cancer research, such as breast cancer, prostate cancer, lung cancer, colorectal cancer, pancreatic esophageal cancer, ovarian cancer, bladder cancer, renal cancer, etc. [10-14]. With the application of modern chromatography and mass spectrometry or other detection techniques like nuclear magnetic resonance, lots of biological metabolites have proven by numbers of statistical methods (such as $t$-test, discriminant analysis, principal component analysis, cluster analysis etc.) to be specific and sensitive biomarkers in current cancer research [10-14]. Recently, some clinical studies showed that biological metabolites in the fluid or tissue samples were of great beneficial in the early diagnosis and managements for GC [15-19]. However, there were still few studies investigated on the serum metabolites might be novel diagnostic indicators for gastrointestinal cancer [20-23].

In this study, we aimed to analyze serum metabolites between GC patients and the healthy people as well as their relationship with the prognosis of GC in order to find potential specific and sensitive serum biomarkers for GC by using high performance liquid chromatography coupled with electrospray ionization/quadrupole-time-offlight mass spectrometry (HPLCESI/Q-TOFMS), which can be of clinical beneficial for the early diagnosis and management of GC.

\section{RESULTS}

\section{Total ion current spectras between GC patients and healthy people in serum samples}

In this study, all the biological metabolites in serum samples from both GC patients and healthy people were detected by using HPLCESI/Q-TOFMS, providing lots of information for clarifying unknown molecular mechanisms for GC. There were obvious difference in the total ion current spectras of serum samples between GC patients and healthy people under the full-scan mode (in retention time of 11 minutes), which suggested that there might be some important metabolic changes in GC patients (Figure 1). By using compared t- test, a total of 87 metabolites in $\mathrm{ESI}^{+}$mode were find to be statistically different between GC patients and healthy people $(p<0.05)$ (Table 1). However, there were no statistically different serum metabolites in $\mathrm{ESI}^{-}$mode between GC patients and healthy people (Figure not shown). The clear identifications of each biological metabolites and their related effect in the biological processes are critical for the metabolomics' researches. Compared with the data obtained from HMDB (www.hmdb.ca) databases, 87 metabolites in $\mathrm{ESI}^{+}$mode were structurally confirmed.

\section{Principal component analysis (PCA)}

PCA was adopted as a statistical tool for clustering the detected serum metabolites into smaller number as principal components (PCs) to find the specific metabolic differences between GC patients and healthy controls in test group (24 GC patients and 24 healthy controls) and then to distinguish the outliers or discretization trends in GC patients. In the current study, almost all the samples were clearly grouped or separated in PCA plots, indicating the serum metabolites were properly classified in GC patients and healthy people (Figure 2).

There were obvious difference between GC patients and healthy people under the full-scan mode, but the response in GC patients or healthy people did not vary a lot. By using two sample $t$-test, a total of 39 metabolites in $\mathrm{ESI}^{+}$mode were found to be statistically different between GC patients and healthy people, which might be of potential power to distinguish the GC patients from the healthy controls $(p<0.05)$ (Table 1$)$.

\section{Partial least squares discriminant analysis (PLS- DA)}

To find the most important serum metabolites between GC patients and healthy controls, partial least squares discriminant analysis (PLS-DA) (Figure 3) was done in test group (24 GC patients and 24 healthy controls). PLS-DA results showed that most of the serum metabolites were clearly clustered in PLS-DA plot with the sensitivity and specificity was $100 \%$. This was consistent with the PCA results, indicating that the 39 serum metabolites could be of statistical importance to separate GC patients and healthy controls. Subsequently, 16 serum metabolites in $\mathrm{ESI}^{+}$mode according to the VIP (Variable Importance in Projection) plots of PLS-DA were found to be highly significant between GC patients and healthy 
volunteers (VIP $>1$ ). Based on above two sample $t$-tests, a hierarchical clustering analysis on the selected serum metabolites was implemented to visualize the relative significant serum metabolites. Statistically, 24 serum metabolites in $\mathrm{ESI}^{+}$mode in test group could definitely distinguish GC patients from healthy peoples (Figure $4 \mathrm{~A}$ ), furthermore, 16 serum metabolites in $\mathrm{ESI}^{+}$mode of VIP $>1$ in validation group could definitely distinguish GC patients from healthy peoples (Figure 4B) $(p<0.05)$.

\section{Relationships between serum metabolites with the clinicopathologic features of GC patients}

According to 16 serum metabolites selected from the PLS-DA results, all the 125 GC patients were divided into 3 groups (Group 1, Group 2 and Group 3) (Figure
5). In the same group of GC patients, responses to the 16 selected serum metabolites were similar. Differences in clinicopathological parameters like tumor differentiation, age, vascular invasion, TNM staging, tumor position and the expression of Ki-67 and P53 were observed among the subgroups by chi-square test (Figure 6). In the GC patients of Group 3, there were significant higher proportion of older patients ( $>60$ years), TNM staging (phase III), poorly differentiated gastric cancer, and upper gastric cancer $(p<0.05)$. In the GC patients of Group 1, there were significant higher proportion of vascular invasion and upper gastric cancer $(p<0.05)$. By using variance analysis, an increased trend in the expression of Ki-67 and P53 was observed among different groups with the highest level in Group $3(p<0.05)$. The KaplanMeier (K-M) survival curve of each group were plotted

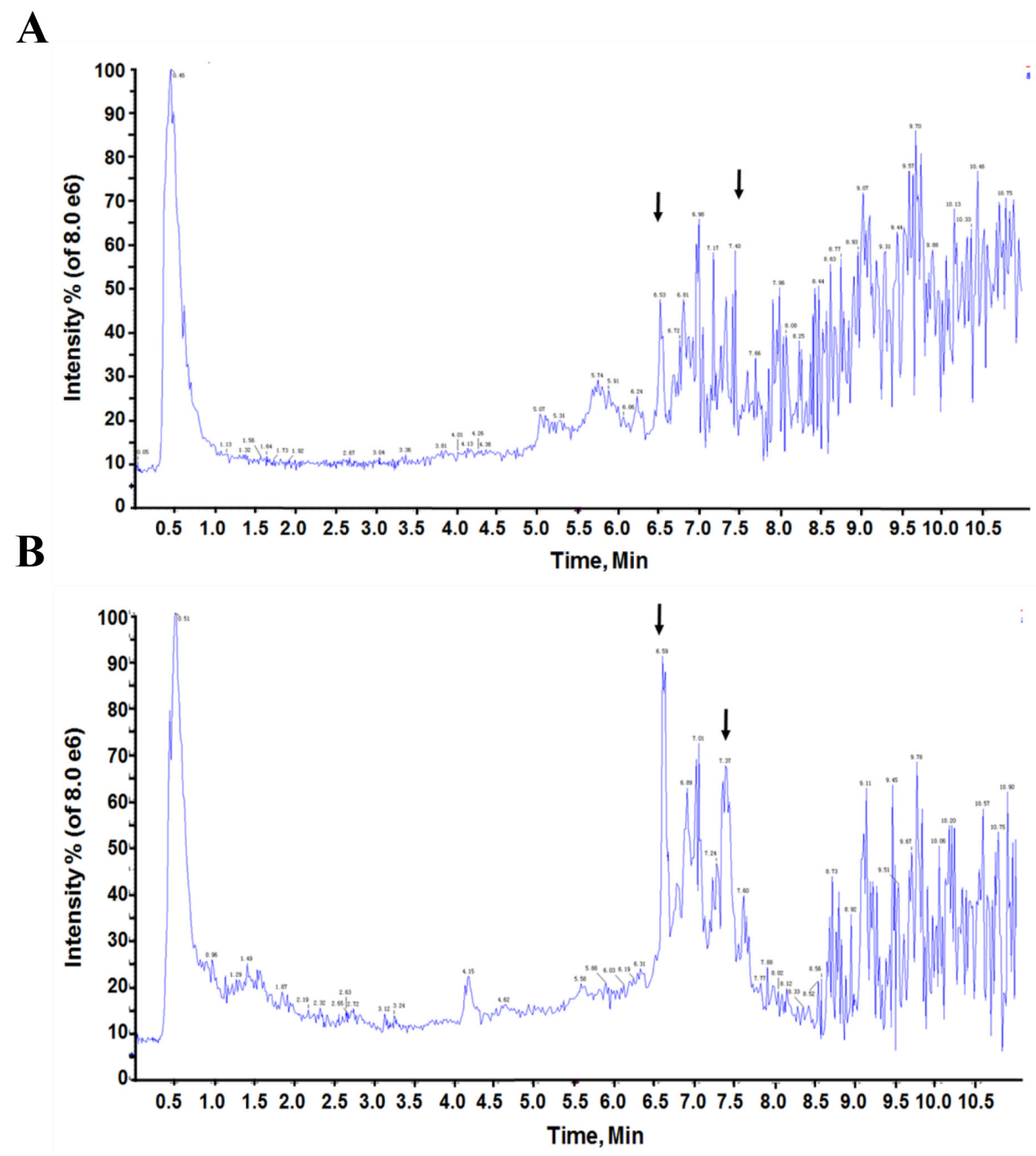

Figure 1: A representative serum total ion current mass spectra of GC patients and healthy people. (A) serum sample of GC patient, (B) serum sample of healthy control. The arrows indicated two markedly different wave crests between A and B. 
Table 1: Biological metabolites identified from serum samples between GC patients and healthy controls by LC-MS/ MS

\begin{tabular}{|c|c|c|c|c|}
\hline No. & $\mathbf{m} / \mathbf{z}$ ratio & Name & $\begin{array}{c}\text { Response in GC } \\
\text { patients }\end{array}$ & $\begin{array}{c}\text { Response in healthy } \\
\text { controls }\end{array}$ \\
\hline 1 & 104.1051 & 3-Aminobutanoic acid & $73.19 \pm 20.12$ & $5.60 \pm 1.34$ \\
\hline 2 & 112.8931 & 2,4-2,4-hexadienoic acid & $56.19 \pm 10.83$ & $4.66 \pm 1.03$ \\
\hline 3 & 224.0947 & Benzoic acid & $400.33 \pm 65.14$ & $31.63 \pm 9.38$ \\
\hline 4 & 246.242 & Valerylcarnitine & $121.88 \pm 41.71$ & $34.26 \pm 23.65$ \\
\hline 5 & 267.6473 & Tetradecylcyclobutanone & $5737.45 \pm 1396.97$ & $1973.43 \pm 249.91$ \\
\hline 6 & 274.2739 & Heptanoylcarnitine & $8842.87 \pm 1862.07$ & $297.34 \pm 63.54$ \\
\hline 7 & 279.6473 & Alpha-Linolenic acid & $3367.18 \pm 687.08$ & $199.02 \pm 59.96$ \\
\hline 8 & 280.2627 & Octanamide & $51.51 \pm 17.31$ & $7.03 \pm 7.03$ \\
\hline 9 & 280.655 & $\mathrm{NA}^{\mathrm{a}}$ & $1328.04 \pm 277.34$ & $28.86 \pm 9.89$ \\
\hline 10 & 282.2789 & Petroselinic acid & $575.40 \pm 240.94$ & $137.69 \pm 46.56$ \\
\hline 11 & 290.2688 & hydroxyhexanoyl carnitine & $106.32 \pm 22.24$ & $27.37 \pm 25.74$ \\
\hline 12 & 291.6468 & Etiocholanolone & $507.15 \pm 133.22$ & $16.10 \pm 9.62$ \\
\hline 13 & 301.1413 & 4-Methoxybenzyl glucoside & $180.99 \pm 43.56$ & $13.19 \pm 3.02$ \\
\hline 14 & 302.3057 & Nonanoylcarnitine & $4585.55 \pm 1731.78$ & $499.35 \pm 189.16$ \\
\hline 15 & 303.2282 & Glycerol tributanoate & $354.91 \pm 57.12$ & $18.69 \pm 7.33$ \\
\hline 16 & 304.2609 & 7-Hydroxyetodolac & $275.46 \pm 87.01$ & $56.67 \pm 26.29$ \\
\hline 17 & 318.3004 & Phytosphingosine & $8913.46 \pm 2027.48$ & $400.52 \pm 94.27$ \\
\hline 18 & 325.2106 & Cibaric acid & $327.67 \pm 91.65$ & $23.10 \pm 5.29$ \\
\hline 19 & 330.3373 & Dihydroceramide & $6873.61 \pm 3931.61$ & $196.00 \pm 58.97$ \\
\hline 20 & 346.3319 & 3-hydroxyundecanoyl carnitine & $1371.12 \pm 402.15$ & $6.53 \pm 6.53$ \\
\hline 21 & 356.2026 & S-Adenosylmethioninamine & $211.11 \pm 84.51$ & $10.12 \pm 3.49$ \\
\hline 22 & 358.3678 & Cyclodopa glucoside & $3736.15 \pm 2409.30$ & $24.56 \pm 6.27$ \\
\hline 23 & 362.3263 & Eicosatrienoic acid isobutylamide & $2214.32 \pm 508.10$ & $28.30 \pm 28.30$ \\
\hline 24 & 374.2451 & 3-hydroxytridecanoyl carnitine & $174.38 \pm 69.82$ & $5.15 \pm 1.06$ \\
\hline 25 & 374.3624 & Pipereicosalidine & $1102.80 \pm 223.45$ & $296.22 \pm 116.53$ \\
\hline 26 & 374.3638 & Benzenediol & $1872.03 \pm 670.08$ & $37.08 \pm 10.08$ \\
\hline 27 & 381.1301 & 3-O-Methylglycyrol & $421.97 \pm 81.57$ & $128.83 \pm 59.19$ \\
\hline 28 & 385.2925 & Persicachrome & $609.10 \pm 145.62$ & $42.08 \pm 10.25$ \\
\hline 29 & 390.358 & $\mathrm{NA}^{\mathrm{a}}$ & $593.58 \pm 104.37$ & $127.06 \pm 35.64$ \\
\hline 30 & 397.1042 & Aloesol 7-glucoside & $596.67 \pm 107.17$ & $64.38 \pm 16.04$ \\
\hline 31 & 398.2422 & PGF2a ethanolamide & $180.03 \pm 63.98$ & $5.27 \pm 1.02$ \\
\hline 32 & 399.3074 & methyltetracosanoic acid & $594.49 \pm 186.36$ & $23.85 \pm 20.64$ \\
\hline 33 & 402.395 & 3-hydroxypentadecanoyl carnitine & $2103.19 \pm 624.11$ & $34.71 \pm 12.66$ \\
\hline 34 & 413.2659 & Abscisic alcohol 11-glucoside & $638.29 \pm 260.28$ & $42.65 \pm 10.10$ \\
\hline 35 & 415.2098 & 4-O-Methylmelleolide & $1673.09 \pm 159.29$ & $680.56 \pm 180.15$ \\
\hline
\end{tabular}

(Continued) 


\begin{tabular}{|c|c|c|c|c|}
\hline No. & $\mathrm{m} / \mathrm{z}$ ratio & Name & $\begin{array}{c}\text { Response in GC } \\
\text { patients }\end{array}$ & $\begin{array}{c}\text { Response in healthy } \\
\text { controls }\end{array}$ \\
\hline 36 & 418.3871 & (E)-Casimiroedine & $107.79 \pm 31.43$ & $5.13 \pm 1.04$ \\
\hline 37 & 429.319 & Sorbitan oleate & $2048.02 \pm 392.91$ & $66.25 \pm 19.67$ \\
\hline 38 & 437.1937 & Phenethyl 6-galloylglucoside & $2428.94 \pm 626.37$ & $1416.72 \pm 296.65$ \\
\hline 39 & 443.334 & 4-carboxylic acid & $585.14 \pm 152.89$ & $47.68 \pm 11.64$ \\
\hline 40 & 453.1674 & Melleolide M & $3263.71 \pm 829.68$ & $65.07 \pm 36.92$ \\
\hline 41 & 457.3499 & $\begin{array}{c}\text { 3-Hydroxycycloart-24-en-21-oic } \\
\text { acid }\end{array}$ & $76.99 \pm 26.06$ & $4.46 \pm 1.00$ \\
\hline 42 & 463.3028 & Lucidenic acid M & $127.09 \pm 48.34$ & $18.37 \pm 14.39$ \\
\hline 43 & 473.3451 & 20beta-Hydroxyursolic acid & $1415.31 \pm 283.51$ & $61.94 \pm 11.68$ \\
\hline 44 & 495.1719 & 3'-O-beta-glucuronide & $546.95 \pm 114.95$ & $84.87 \pm 84.87$ \\
\hline 45 & 508.2495 & 2-Decarboxybetanin & $120.77 \pm 39.00$ & $34.96 \pm 8.12$ \\
\hline 46 & 523.2461 & Physangulide & $378.89 \pm 96.94$ & $47.19 \pm 9.89$ \\
\hline 47 & 545.26 & Cinncassiol A 19-glucoside & $701.86 \pm 144.20$ & $74.31 \pm 13.06$ \\
\hline 48 & 567.2735 & Hordatine A & $1346.25 \pm 237.43$ & $208.17 \pm 50.16$ \\
\hline 49 & 568.4302 & Neoacrimarine $\mathrm{H}$ & $1889.75 \pm 284.80$ & $275.31 \pm 48.47$ \\
\hline 50 & 574.2894 & Biotinyl-5'-AMP & $390.73 \pm 167.63$ & $24.03 \pm 24.03$ \\
\hline 51 & 580.2921 & $\begin{array}{l}\text { Pelargonidin 3-rhamnoside } \\
\text { 5-glucoside }\end{array}$ & $4141.02 \pm 987.75$ & $27.99 \pm 20.16$ \\
\hline 52 & 585.2696 & Bilirubin & $734.33 \pm 204.06$ & $150.44 \pm 68.99$ \\
\hline 53 & 589.2874 & D-Urobilin & $1928.48 \pm 327.89$ & $182.80 \pm 35.41$ \\
\hline 54 & 590.4269 & $\mathrm{NA}^{\mathrm{a}}$ & $758.58 \pm 209.97$ & $50.96 \pm 13.75$ \\
\hline 55 & 601.2987 & Gluten exorphin & $780.67 \pm 190.11$ & $36.48 \pm 8.94$ \\
\hline 56 & 604.2921 & Neocasomorphin & $1302.89 \pm 255.42$ & $81.58 \pm 24.79$ \\
\hline 57 & 606.3082 & Amphibine $\mathrm{H}$ & $1014.17 \pm 188.93$ & $125.36 \pm 17.64$ \\
\hline 58 & 611.3 & Endomorphin & $2765.37 \pm 432.30$ & $104.87 \pm 67.11$ \\
\hline 59 & 633.3133 & Coagulin R 3-glucoside & $4175.29 \pm 640.38$ & $138.07 \pm 123.68$ \\
\hline 60 & 637.4743 & 9-Oxoasimicinone & $1147.96 \pm 314.53$ & $156.41 \pm 32.71$ \\
\hline 61 & 638.2497 & Heparan sulfate & $666.21 \pm 132.75$ & $84.87 \pm 19.96$ \\
\hline 62 & 648.2786 & Indoleacetyl glutamine & $729.88 \pm 239.73$ & $28.15 \pm 6.35$ \\
\hline 63 & 648.3096 & Nummularine A & $1444.89 \pm 267.22$ & $42.69 \pm 8.35$ \\
\hline 64 & 659.2882 & Gluten exorphin & $9057.13 \pm 1693.18$ & $120.98 \pm 38.72$ \\
\hline 65 & 663.3058 & Physalolactone B 3-glucoside & $1128.26 \pm 309.07$ & $44.65 \pm 15.35$ \\
\hline 66 & 669.4128 & Isolimonic acid glucoside & $537.72 \pm 173.15$ & $26.31 \pm 20.60$ \\
\hline 67 & 670.3232 & Nummularine B & $2960.59 \pm 526.46$ & $62.81 \pm 7.90$ \\
\hline 68 & 677.3397 & Cucurbitacin I 2-glucoside & $7285.01 \pm 1103.93$ & $270.65 \pm 53.18$ \\
\hline 69 & 681.8775 & Omphalotin A & $505.93 \pm 151.78$ & $28.23 \pm 6.85$ \\
\hline 70 & 685.3196 & Neoacrimarine B & $1139.76 \pm 344.13$ & $29.83 \pm 8.00$ \\
\hline
\end{tabular}

(Continued) 


\begin{tabular}{lcccc}
\hline No. & m/z ratio & Name & $\begin{array}{c}\text { Response in GC } \\
\text { patients }\end{array}$ & $\begin{array}{c}\text { Response in healthy } \\
\text { controls }\end{array}$ \\
\hline 71 & 692.3353 & Alpha-Tetrasaccharide & $4325.35 \pm 671.19$ & $231.51 \pm 54.84$ \\
72 & 699.3534 & Corchorusoside A & $7137.84 \pm 1140.89$ & $169.75 \pm 63.62$ \\
73 & 700.2265 & Pteroyltriglutamic acid & $550.30 \pm 163.99$ & $19.08 \pm 16.40$ \\
74 & Fasciculic acid C & $745.48 \pm 204.58$ & $21.68 \pm 21.68$ \\
75 & 710.2494 & Delphinidin 3-5-glucoside & $4938.62 \pm 839.57$ & $85.83 \pm 37.91$ \\
76 & 714.3493 & Glutamate & $282.12 \pm 133.46$ & $24.85 \pm 19.14$ \\
77 & 718.4179 & PG & $8637.88 \pm 1248.68$ & $185.68 \pm 34.10$ \\
78 & 721.3659 & Hordatine B glucoside & $7222.37 \pm 1169.08$ & $160.23 \pm 29.39$ \\
79 & 743.3773 & 28-Glucosyloleanolic acid & $2299.14 \pm 501.59$ & $378.22 \pm 96.16$ \\
80 & 751.3585 & 3-sambubioside 5-glucoside & $6708.47 \pm 1087.93$ & $410.25 \pm 85.23$ \\
81 & 758.3772 & Momordin I & $8359.28 \pm 1443.52$ & $856.41 \pm 100.21$ \\
82 & 765.3931 & NA & $3911.48 \pm 1009.33$ & $412.08 \pm 85.32$ \\
83 & 806.9407 & Sulfogalactosylceramide & $4608.88 \pm 792.23$ & $210.96 \pm 118.04$ \\
84 & 836.5099 & Hovenidulcioside A1 & $2695.84 \pm 650.38$ & $282.23 \pm 85.97$ \\
85 & 853.443 & Pectenotoxin & $1639.73 \pm 515.89$ & $69.48 \pm 10.31$ \\
86 & 875.4587 & Isohopeaphenol & $473.66 \pm 271.60$ & $17.46 \pm 3.42$ \\
87 & 907.3786 & (3S)-3-Hydroxyadipyl-CoA & $1326.56 \pm 333.41$ & $76.58 \pm 8.54$ \\
\hline
\end{tabular}

Note: Values of response were expressed as mean \pm SD. NA ${ }^{a}$ : not available.

and the survival rates were computed correspondingly (Figure 5). Based on the Kaplan-Meier (K-M) survival curve, survival rate statistically vary a lot among the three groups and significant differences in survival time were obviously observed between Group 1 and Groups 2 or Group 3(p<0.05) (Figure 7).

\section{Relationships between serum metabolites with the prognosis of GC}

As analyzed by univariate analysis of 87 serum metabolites in $\mathrm{ESI}^{+}$mode from all the $125 \mathrm{GC}$ patients in this study, prognostic factors for GC consisted of 2,4-hexadienoic acid, 4-methylphenyl dodecanoate, glycerol tributanoate, methionyl-methionine and PG $(p<0.05)$ (Table 3). Subsequently, multivariate COX regression analysis, variance analysis and the $\mathrm{K}-\mathrm{M}$ survival curve were used to find the independent prognostic factor for GC in human serum. Multivariate COX regression analysis showed TNM staging $(p<0.005,95 \%$ confidence interval $(95 \% \mathrm{CI})$ 1.002-1.034), 2,4-hexadienoic acid $(p<0.005, \quad 95 \% \quad$ CI $1.002-1.027), \quad 4$-methylphenyl dodecanoate $(p<0.005,95 \% 95 \%$ CI $1.002-1.001)$ and glycerol tributanoate $(p<0.005,95 \%$ CI $1.002-1.032)$ were independent prognostic factors of GC (Table 4). In variance analysis, the 3 selected serum metabolites (2,4-hexadienoic acid, 4-methylphenyl dodecanoate and glycerol tributanoate) in $\mathrm{ESI}^{+}$mode in both high level group and low level group could definitely distinguish GC patients from healthy peoples (Figure 8$)(p<0.05)$. As shown in the K-M survival curve, the survival rate in high level group of the 3 selected serum metabolites (2,4-hexadienoic acid, 4-methylphenyl dodecanoate and glycerol tributanoate) together was significant lower than in those in low level group of the 3 selected serum metabolites (Figure 9) $(p<0.05)$. Consistent with the the K-M survival result with only 1 selected serum metabolite (2,4-hexadienoic acid, 4-methylphenyl dodecanoate or glycerol tributanoate alone) (Figure 9) $(p<0.05)$, this might suggest that the 3 selected serum metabolites (2,4-hexadienoic acid, 4-methylphenyl dodecanoate and glycerol tributanoate) can be considered to be useful prognostic factors for GC.

\section{DISCUSSION}

More and more biological studies showed that biological metabolites were "downstream" to genes or "endpoint markers" for disease, and thereby metabolomics is now of global attentions in cancer research in the field of breast cancer, prostate cancer, lung cancer, colorectal cancer, pancreatic esophageal cancer, ovarian cancer, bladder cancer and renal cancer for early diagnosis and effective managements as well 
as molecular mechanisms. Detected by using modern chromatography and mass spectrometry or other detection techniques, lots of biological metabolites have proven by numbers of statistical methods (such as $t$-test, partial least squares discriminant analysis, principal component analysis, cluster analysis, etc.) to be potential specific and sensitive biomarkers for different cancer [10-14]. Developed from the malignant cells in the stomach inner lining, gastric cancer (GC) is a major cause of cancer-related death today. Traditional diagnosis methods consist of biopsy, endoscopy and pathological examination [1-3]. However, these diagnosis methods involve with significant limitations, and the efficiency was inconsistent among different endoscopists and pathologists. Recently, some clinical studies showed that biological metabolites in the fluid or tissue samples were of great beneficial in the early diagnosis and managements for GC [15-19]. Whereas, there were still few studies recommended using serum metabolites as a novel diagnostic approach for GC [20-23]. In this study, serum metabolites were investigated between gastric cancer (GC) patients and the healthy people as well as their relationships with the prognosis of GC in order to find potential specific serum biomarkers for GC by using high performance liquid chromatography coupled with electrospray ionization/quadrupole-time-of-flight mass spectrometry (HPLCESI/Q-TOFMS). Statistically, a total of 87 metabolites (Table 1) in ESI+ mode were find to be statistically different between GC patients and healthy people, including 16 serum metabolites in ESI+ mode of VIP $>1$ in both test group and validation group which could definitely distinguish GC patients from healthy peoples $(p<0.05)$ (Figure 4). According to 16 serum metabolites selected from the PLS-DA results (Table 2 ), all the 125 GC patients were divided into 3 groups (Group 1, Group 2 and Group 3) (Figure 5). Serum metabolites detected in this study with statistical different responses in between GC patients and healthy people reveal several important metabolic or molecular pathways for GC. Firstly, most gastric cancer cells produce energy primarily through Valsalva effect instead of the citric acid cycle and will change the serum levels of the metabolites of the citric acid cycles [21]. Secondly, the disorder of serum amino acid can influence the cell growth, cell

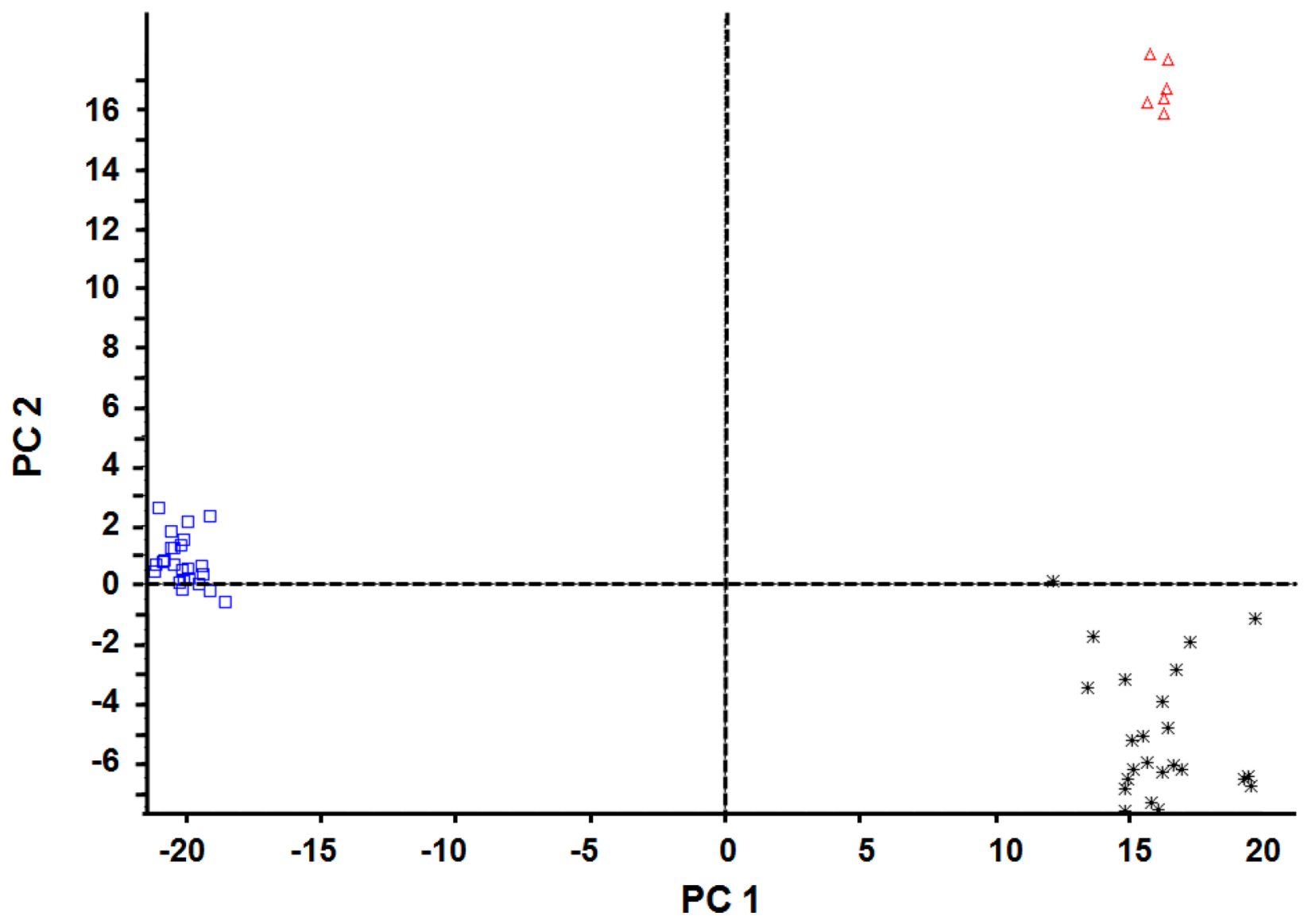

Figure 2: Principal component analysis (PCA) scores plots of serum biological metabolites from GC patients and healthy people in test group in ESI+ mode. Blank square ( $\square)$, healthy people, 24 samples; black star $\left(^{*}\right)$, GC patients, 24 samples; blank triangle $(\Delta)$, quality control, 6 samples. ESI: electrospray ionization. 
A

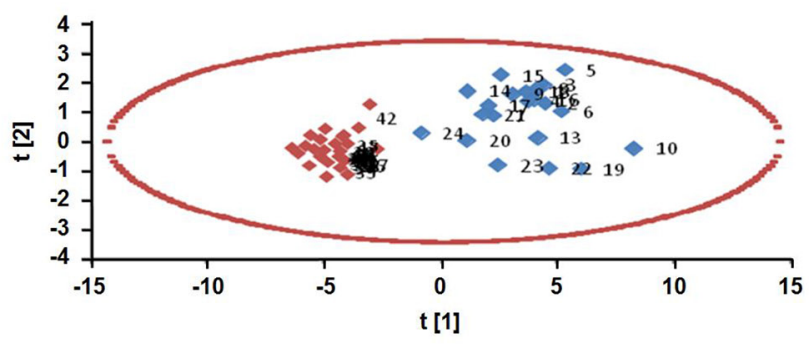

C

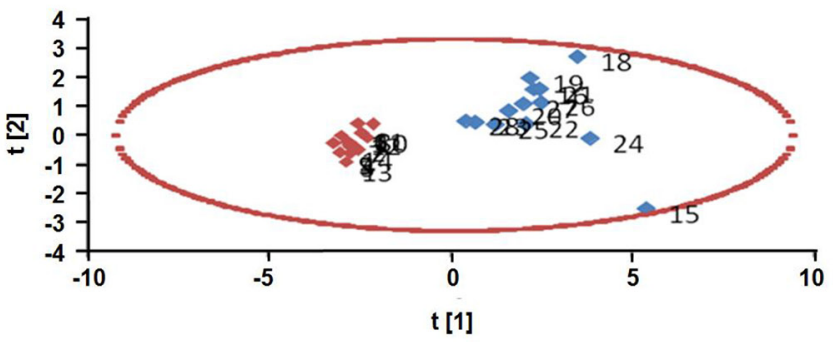

B

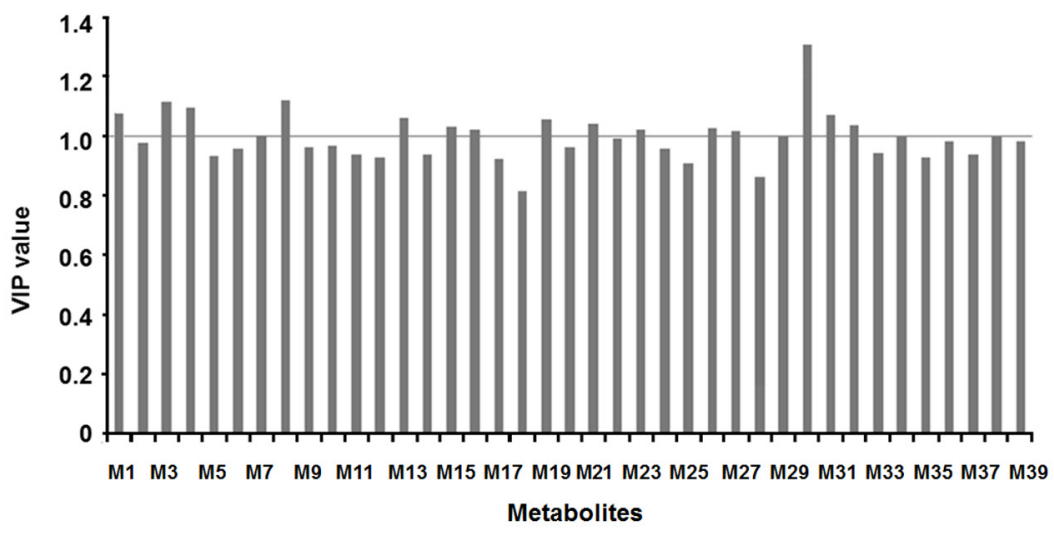

Figure 3: Partial least squares discriminant analysis (PLS-DA) of serum biological metabolites of GC patients and healthy people in ESI+ mode. (A) PLS-DA score plots in the test group, Red, healthy controls; blue, GC patients; (B) VIP plots of PLS-DA analysis in the test group, the line in the figure indicate VIP=1, M1-M39 is the number of 39 metabolites in the Table 2; (C) PLSDA score plots in the validation group. Red, healthy controls; blue, GC patients. ESI: electrospray ionization.

A

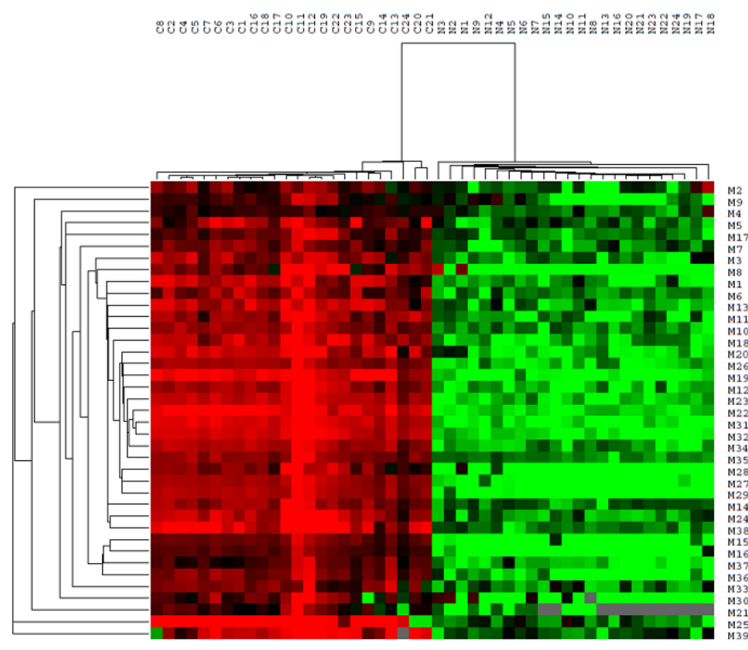

B

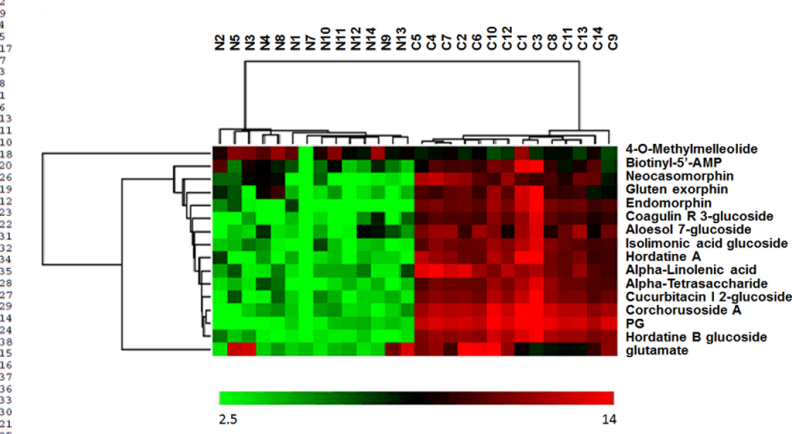

Figure 4: Hierarchical clustering analysis of serum biological metabolites of GC patients and healthy people in ESI ${ }^{+}$mode in the test group (24 GC patients and 24 healthy controls, 39 serum metabolites determined by PCA) (A) and in the validation group (14 GC patients and 14 healthy control, 16 serum metabolites determined by PLS-DA) (B). Red, positive value; green, negative value; black, equal to zero. Each GC patient or healthy people was listed in column. Each serum biological metabolite was listed in row. N: healthy controls; T: GC patients. ESI: electrospray ionization. M1-M39 is the No. of 39 metabolites in the Table 2. 
metastasis and cell apoptosis as the raw materials for the protein and nucleic acid synthesis of the cancer cells [24]. Additionally, the disorder of serum fatty acid can also affect the cell growth, cell metastasis and cell apoptosis as well as tumor angiogenesis through untaken or over-exploited by the cancer cell proliferation and growth, or inhibited by the synthesis [9]. Furthermore, other substances like lactate, creatine and succinate were also involved in the metabolic pathways in GC patients comparing with the healthy controls [25].
Clinicopathological parameters like tumor differentiation, age, vascular invasion, TNM staging, survival rate, tumor position and the expression of Ki-67 and P53 were statistically different among the subgroups divided by the 16 serum metabolites selected from the PLS-DA results by the using chi-square test or KaplanMeier $(\mathrm{K}-\mathrm{M})$ survival curve $(\mathrm{p}<0.05)$ (Figure 6$)$. These results was consistent with some prvious studies showing some structural proteins include receptors, membrane channel proteins and enzymes like SRY (sex determining

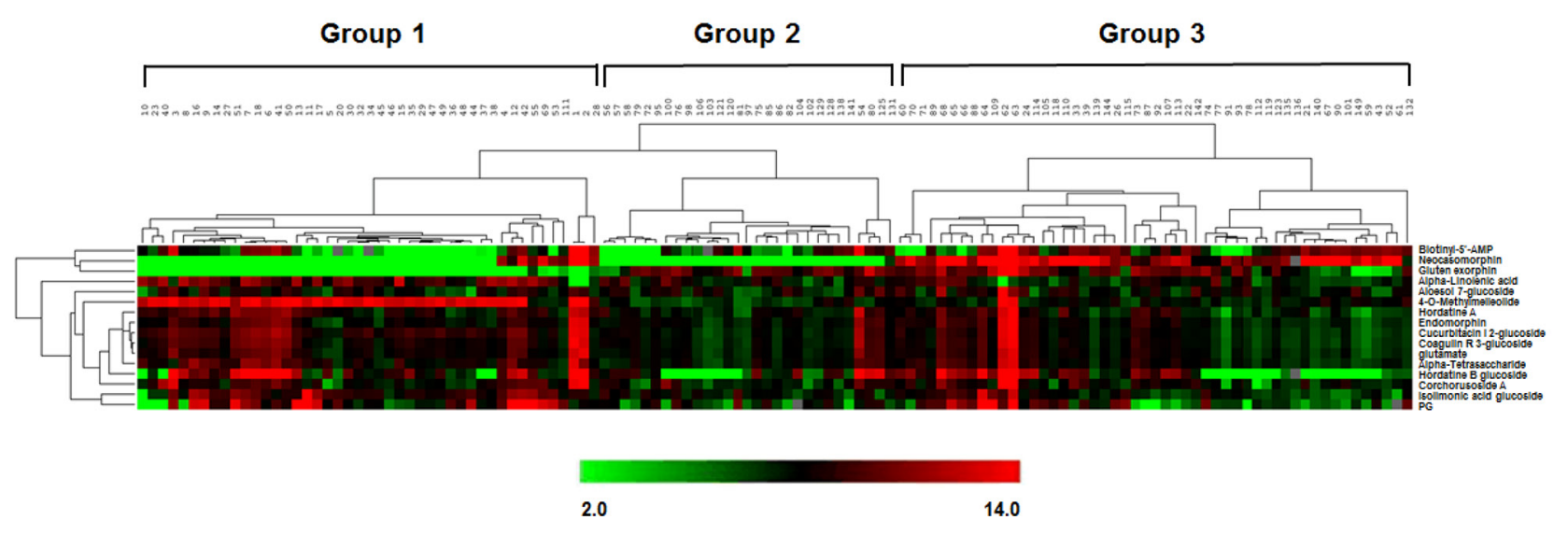

Figure 5: Hierarchical clustering analysis of the 16 selected serum metabolites in ESI+ mode of all the 125 GC patients in this study. Red, positive value; green, negative value; black, equal to zero. In the same group of GC patients, responses to the 16 selected serum metabolites were similar. N: healthy controls; T: GC patients. ESI: electrospray ionization.

A

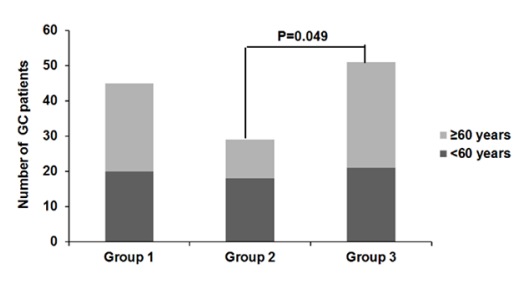

D
B

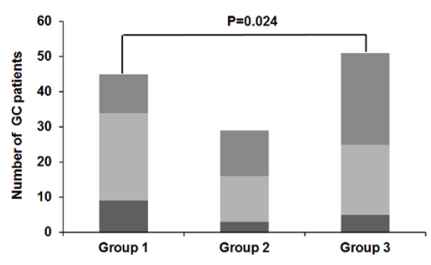

C

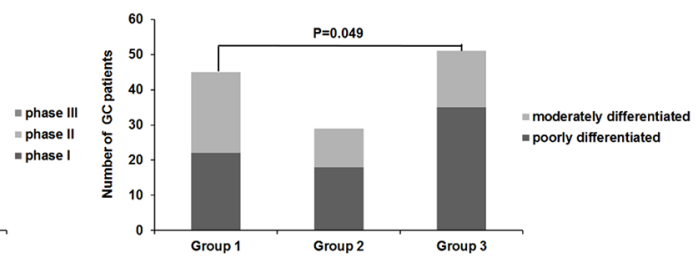

$\mathbf{E}$
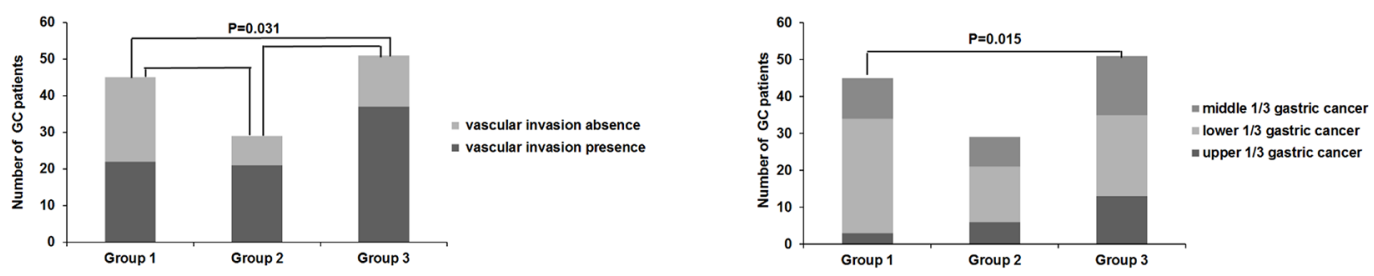

Figure 6: Histograms of different clinicopathologic features of all the 125 GC patients among the 3 groups divided by the 16 selected serum metabolites in ESI+ mode. (A) Proportion of different age ( $>60$ years and $<60$ years), (B) Proportion of different TNM staging (phase I, phase II and phase III); (C) Proportion of different differentiation type (moderate differentiation and poor differentiation); (D) Proportion of different vascular invasion type (presence and absence; (E) Proportion of different tumor position (upper gastric cancer, antrum gastric cancer and lower gastric cancer). ESI: electrospray ionization. 
region Y)-box 2, serum gastrin, pepsinogen I and octamerbinding protein-4 (OCT4) plays a vital role in gastric cancer metastasis or differentiation and thereby in TNM staging of GC [26, 27]. Ki-67 and and P53 were highly expressed in GC, and factors influence the expression of Ki-67 and P53 might be important for regulating the cell growth, cell metastasis and cell apoptosis in GC [28].

Currently, serum metabolites like cyclin-dependent kinase 2 (Cdk2), Protein kinase B (Akt) and oleic acid were recommended to be independent prognostic factors of GC $[18,29]$. In this metabolomics study, univariate analysis showed that 2,4-hexadienoic acid, 4-methylphenyl dodecanoate, glycerol tributanoate, methionyl-methionine and PG might be the prognostic factors for GC (Table 3). However, TNM staging ( $p<0.005,95 \%$ confidence interval (95\% CI) 1.002-1.034), 2,4-hexadienoic acid $(p<0.005$,
95\% CI 1.002-1.027), 4-methylphenyl dodecanoate $(p<0.005,95 \%$ CI 1.002-1.001) and glycerol tributanoate $(p<0.005,95 \%$ CI 1.002-1.032) were subsequently confirmed to be independent prognostic factors of $\mathrm{GC}$, by using multivariate COX regression analysis (Table 4). Additionally, in the K-M survival analysis, the survival rate in high level group of the 3 selected serum metabolites together or alone was significant lower than in those in low level group $(p<0.05)$ (Figure 9$)$. All of the 3 selected serum metabolites (2,4-hexadienoic acid, 4-methylphenyl dodecanoate and glycerol tributanoate ) were fatty acid, and high level of the these metabolites in GC patients might be related with the high level of fatty acid synthase regulated by sterol regulatory element-binding proteins (SREBPs) in the fatty acid pathway in GC [30, 31]. All these results might indicate that low serum levels of

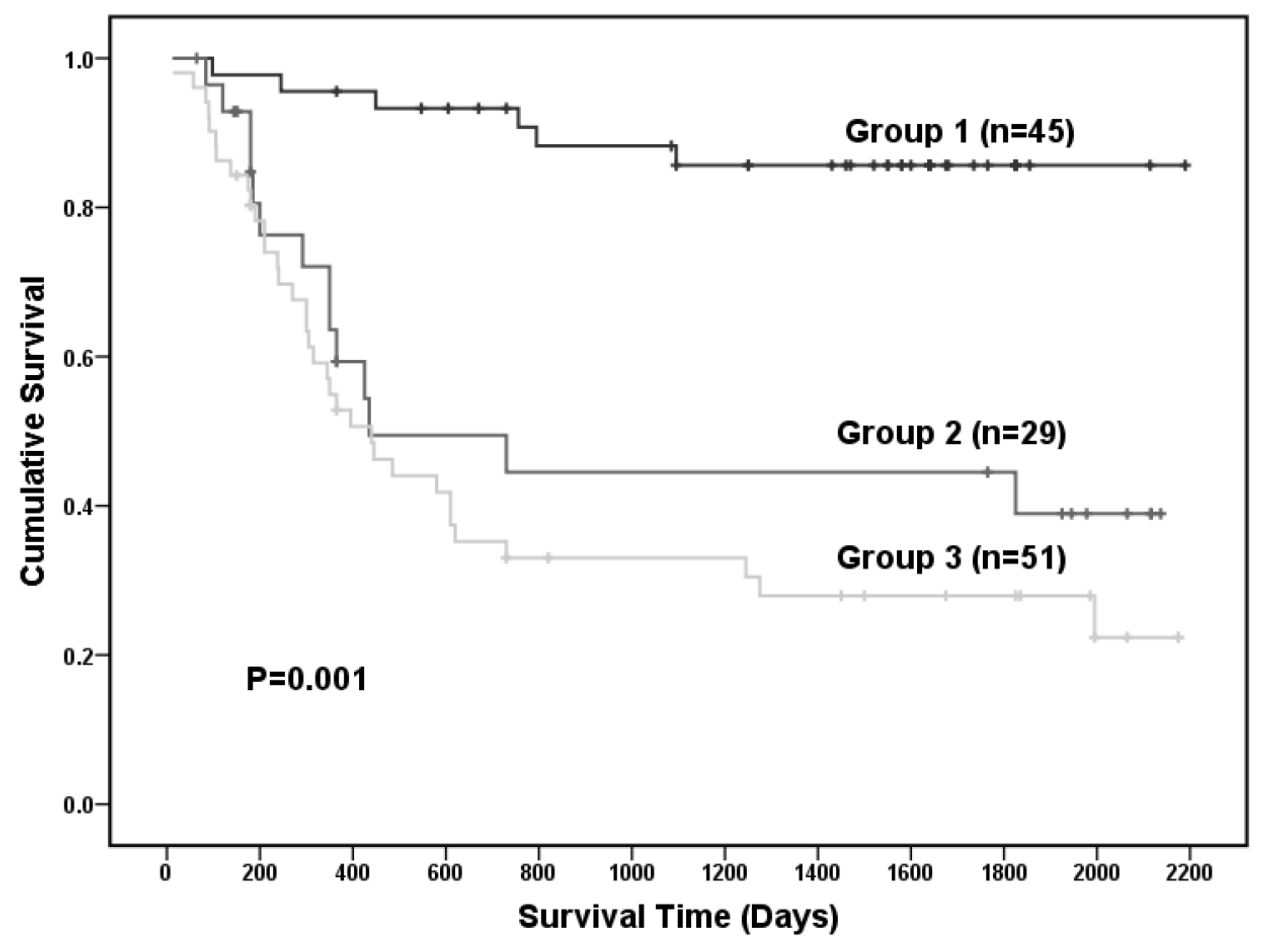

Figure 7: The Kaplan-Meier (K-M) survival curve of all the 125 GC patients among the different groups by the 16 selected serum metabolites in ESI+ mode.

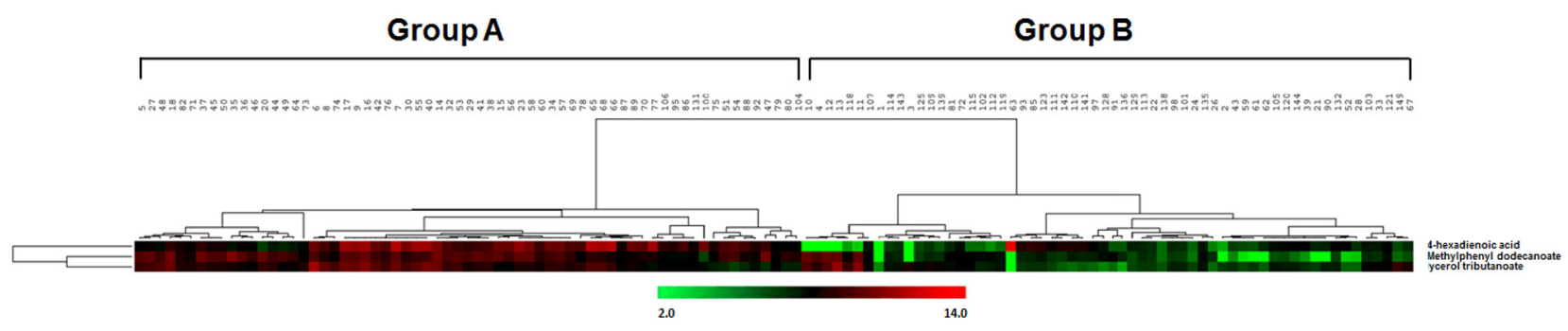

Figure 8: Hierarchical clustering analysis of the 3 selected serum metabolites of all the 125 GC patients in this study. Group A, high response level; Group B, low response level. ESI: electrospray ionization. 
2,4-hexadienoic acid, 4-methylphenyl dodecanoate and glycerol tributanoate may be independent prognostic factors of GC.

In the current study, there are some limitations. Firstly, more clinicopathological parameters like gender, weight, BMI, eating habits or other biomarkers for GC like related miRNA or RNA levels like let-7, matrix metalloproteinase levels like MMP-3, MMP7 and MMP13, COX-2 levels should also be observed. Secondly, the groups should also into more detailed subgroups for each clinicopathological parameters. Finally, other online compound databanks besides HMDB, including METLIN, LIPID MAPS and CEU Mass Mediator, should be used to confirm the chemical structure of the serum metabolites. All these limitations might cause some variation to the results.

To conclude, with the application of modern chromatography and detection techniques as well as different statistical methods, genomics, transcriptomics, proteomics and metabolomics is of key importance in biological studies, especially in the fields of establishing specific potential biomarkers for early diagnosis and effective managements or finding novel molecular mechanisms for the cell growth, cell metastasis and cell apoptosis, tumor angiogenesis in current cancer research, such as breast cancer, prostate cancer, lung cancer, colorectal cancer, pancreatic esophageal cancer, ovarian cancer, bladder cancer, renal cancer, etc. More and more biological studies showed that biological metabolites were highly related with clinicopathological parameters like tumor differentiation, age, vascular invasion, TNM staging, survival rate, tumor position, as well as the prognosis of the cancer. In this metabolomics study, 16 serum metabolites was found to be able to distinguish the GC patients from the healthy controls and 3 serum metabolites (2,4-hexadienoic acid, 4-methylphenyl dodecanoate and glycerol tributanoate) of fatty acid pathways may be independent prognostic factors of GC,
A

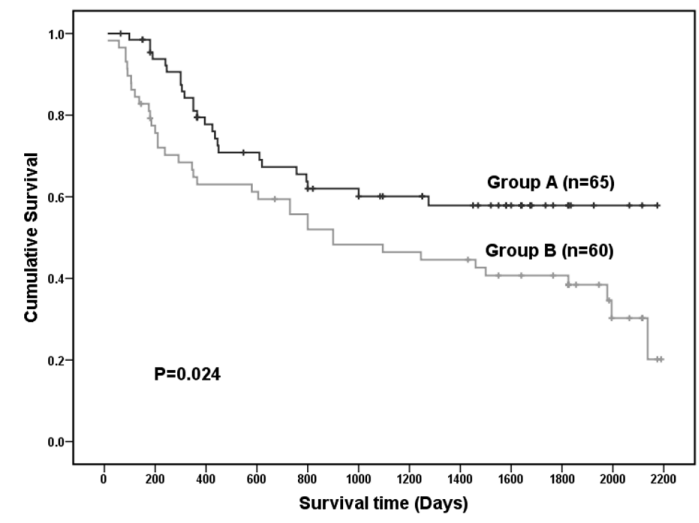

C

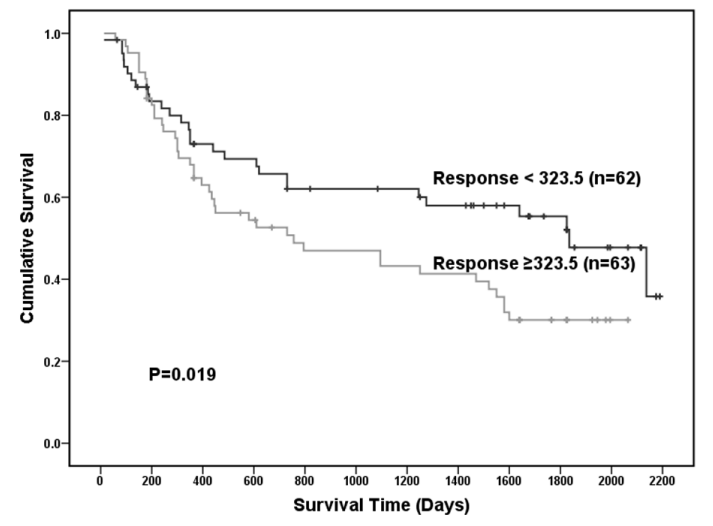

B

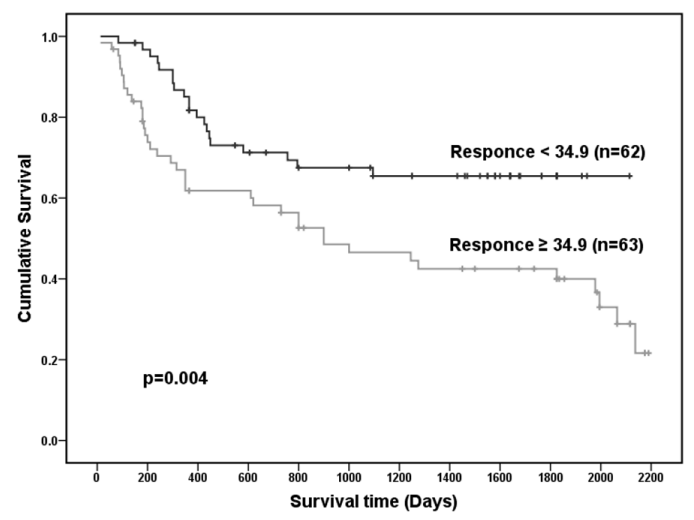

D

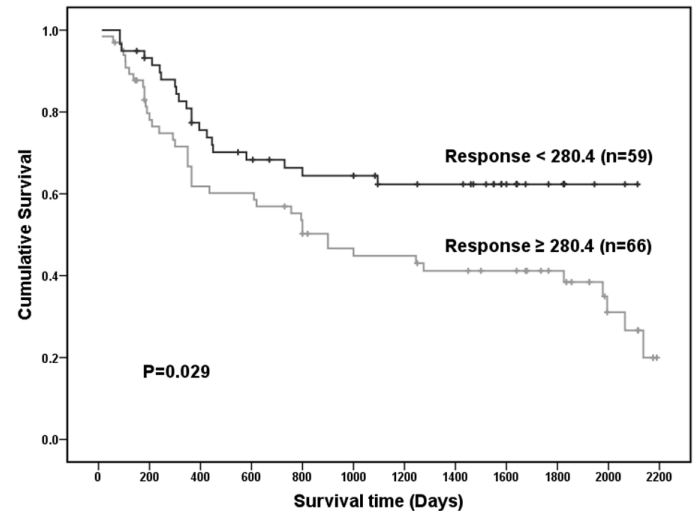

Figure 9: The Kaplan-Meier (K-M) survival curve of all the 125 GC patients among the different groups by the 3 selected serum metabolites in ESI+ mode (2,4-hexadienoic acid, 4-methylphenyl dodecanoate and glycerol tributanoate). (A) $\mathrm{K}-\mathrm{M}$ survival curve of among the different groups by the 3 selected serum metabolites (2,4-hexadienoic acid, 4-methylphenyl dodecanoate and glycerol tributanoate) together; (B) K-M survival curve of among the different groups by 2,4-hexadienoic acid only; (C) K-M survival curve of among the different groups by 4-methylphenyl dodecanoate only; (D) K-M survival curve of among the different groups by glycerol tributanoate only. ESI: electrospray ionization. 
Table 2: Biological metabolites identified from serum samples of GC patients by LC-MS/MS

\begin{tabular}{|c|c|c|c|c|}
\hline No. & $\mathrm{m} / \mathrm{z}$ ratio & Name & Response & VIP value \\
\hline M1 & 279.6473 & Alpha-Linolenic acid & $3367.18 \pm 687.08$ & 1.077265 \\
\hline M2 & 381.1301 & 3-O-Methylglycyrol & $421.97 \pm 81.57$ & 0.976792 \\
\hline M3 & 397.1042 & Aloesol 7-glucoside & $596.67 \pm 107.17$ & 1.114385 \\
\hline M4 & 415.2098 & 4-O-Methylmelleolide & $1673.09 \pm 159.29$ & 1.09627 \\
\hline M5 & 523.2461 & Physangulide & $378.89 \pm 96.94$ & 0.933432 \\
\hline M6 & 545.26 & Cinncassiol A 19-glucoside & $701.86 \pm 144.20$ & 0.956085 \\
\hline M7 & 567.2735 & Hordatine A & $1346.25 \pm 237.43$ & 1.001599 \\
\hline M8 & 574.2894 & Biotinyl-5'-AMP & $390.73 \pm 167.63$ & 1.120802 \\
\hline M9 & 585.2696 & Bilirubin & $734.33 \pm 204.06$ & 0.964372 \\
\hline M10 & 589.2874 & D-urobilin & $1928.48 \pm 327.89$ & 0.964866 \\
\hline M11 & 590.4269 & $\mathrm{NA}^{\mathrm{a}}$ & $758.58 \pm 209.97$ & 0.936668 \\
\hline M12 & 601.2987 & Gluten exorphin & $780.67 \pm 190.11$ & 0.926127 \\
\hline M13 & 604.2921 & Neocasomorphin & $1302.89 \pm 255.42$ & 1.059444 \\
\hline M14 & 606.3082 & Amphibine $\mathrm{H}$ & $1014.17 \pm 188.93$ & 0.93589 \\
\hline M15 & 611.3 & Endomorphin & $2765.37 \pm 432.30$ & 1.03234 \\
\hline M16 & 633.3133 & Coagulin R 3-glucoside & $4175.29 \pm 640.38$ & 1.020049 \\
\hline M17 & 637.4743 & 9-Oxoasimicinone & $1147.96 \pm 314.53$ & 0.924135 \\
\hline M18 & 648.2786 & Indoleacetyl glutamine & $729.88 \pm 239.73$ & 0.814445 \\
\hline M19 & 659.2882 & Gluten exorphin & $9057.13 \pm 1693.18$ & 1.057791 \\
\hline M20 & 663.3058 & Physalolactone B 3-glucoside & $1128.26 \pm 309.07$ & 0.96284 \\
\hline M21 & 669.4128 & Isolimonic acid glucoside & $537.72 \pm 173.15$ & 1.039349 \\
\hline M22 & 670.3232 & Nummularine B & $2960.59 \pm 526.46$ & 0.990336 \\
\hline M23 & 677.3397 & Cucurbitacin I 2-glucoside & $7285.01 \pm 1103.93$ & 1.020499 \\
\hline M24 & 681.8775 & Omphalotin A & $505.93 \pm 151.78$ & 0.958896 \\
\hline M25 & 685.3196 & Neoacrimarine B & $1139.76 \pm 344.13$ & 0.906009 \\
\hline M26 & 692.3353 & Alpha-Tetrasaccharide & $4325.35 \pm 671.19$ & 1.024838 \\
\hline M27 & 699.3534 & Corchorusoside A & $7137.84 \pm 1140.89$ & 1.018284 \\
\hline M28 & 710.2494 & Fasciculic acid $\mathrm{C}$ & $745.48 \pm 204.58$ & 0.856801 \\
\hline M29 & 714.3493 & Delphinidin 3-5-glucoside & $4938.62 \pm 839.57$ & 0.996077 \\
\hline M30 & 718.4179 & Glutamate & $282.12 \pm 133.46$ & 1.307308 \\
\hline M31 & 721.3659 & PG & $8637.88 \pm 1248.68$ & 1.07165 \\
\hline M32 & 743.3773 & Hordatine B glucoside & $7222.37 \pm 1169.08$ & 1.036024 \\
\hline M33 & 751.3585 & 28-Glucosyloleanolic acid & $2299.14 \pm 501.59$ & 0.943374 \\
\hline M34 & 758.3772 & 3-sambubioside 5-glucoside & $6708.47 \pm 1087.93$ & 0.997932 \\
\hline M35 & 765.3931 & Momordin I & $8359.28 \pm 1443.52$ & 0.928922 \\
\hline M36 & 836.5099 & Sulfogalactosylceramide & $4608.88 \pm 792.23$ & 0.980611 \\
\hline M37 & 853.443 & Hovenidulcioside A1 & $2695.84 \pm 650.38$ & 0.936904 \\
\hline M38 & 875.4587 & Pectenotoxin & $1639.73 \pm 515.89$ & 0.997788 \\
\hline M39 & 907.3786 & Isohopeaphenol & $473.66 \pm 271.60$ & 0.980952 \\
\hline
\end{tabular}

Note: Values of response were expressed as mean \pm SD. NA a : not available. VIP: Variable Importance in Projection 
Table 3: Univariate analysis of 87 serum metabolites from all the 125 GC patients in this study

\begin{tabular}{lllllcr}
\hline Variables & B & SE & P value & OR & \multicolumn{2}{c}{ 95.0\% CI for OR } \\
\cline { 3 - 6 } & & & & Lower & Upper \\
\hline 2,4-hexadienoic acid & 0.017 & 0.005 & $0.002^{*}$ & 1.017 & 1.006 & 1.027 \\
4-Methylphenyl dodecanoate & 0.000 & 0.000 & $0.013^{*}$ & 1.000 & 1.000 & 1.000 \\
Glycerol tributanoate & 0.001 & 0.000 & $0.048^{*}$ & 1.001 & 1.001 \\
Methionyl-Methionine & 0.002 & 0.001 & $0.004^{*}$ & 1.002 & 1.001 & 1.003 \\
PG & 0.001 & 0.000 & $0.032^{*}$ & 1.001 & 1.000 & 1.001 \\
\hline
\end{tabular}

Note: ${ }^{*} p<0.05$ was set to be of statistical significance.

Table 4: Multivariate COX regression analysis to find independent prognostic factors of GC

\begin{tabular}{|c|c|c|c|c|c|c|}
\hline \multirow[t]{2}{*}{ Variables } & \multirow[t]{2}{*}{ B } & \multirow[t]{2}{*}{ SE } & \multirow[t]{2}{*}{ P value } & \multirow[t]{2}{*}{ OR } & \multicolumn{2}{|c|}{$95.0 \%$ CI for OR } \\
\hline & & & & & Lower & Upper \\
\hline Gender & 0.028 & 0.307 & 0.928 & 1.028 & 0.563 & 1.878 \\
\hline Age & 0.008 & 0.012 & 0.493 & 1.008 & 0.985 & 1.031 \\
\hline Vascular invasion & 0.293 & 0.287 & 0.308 & 1.340 & 0.763 & 2.352 \\
\hline TNM staging & 0.018 & 0.008 & $0.024^{*}$ & 1.018 & 1.002 & 1.034 \\
\hline Tumor differentiation & -0.112 & 0.286 & 0.696 & 0.894 & 0.511 & 1.565 \\
\hline 2,4-hexadienoic acid & 0.016 & 0.008 & $0.025^{*}$ & 1.016 & 1.002 & 1.027 \\
\hline 4-Methylphenyl dodecanoate & 0.001 & 0.001 & $0.001^{*}$ & 1.001 & 1.000 & 1.001 \\
\hline Glycerol tributanoate & 0.017 & 0.008 & $0.023^{*}$ & 1.017 & 1.002 & 1.032 \\
\hline
\end{tabular}

Note: ${ }^{*} p<0.05$ was set to be of statistical significance.

which might be of great beneficial for the early diagnosis and management of GC. To conclude, low serum levels of 2,4-hexadienoic acid, 4-methylphenyl dodecanoate and glycerol tributanoate may be important independent prognostic factors of GC.

\section{MATERIALS AND METHODS}

\section{Study design}

Blood samples of 125 GC patients of unifocal GC at initial stage and 38 healthy people recruited in our hospital from September 2008 to August 2009 were analyzed in this study. The blood samples of all the patients and healthy people were extracted in the morning and the basal metabolic rate (BMR) were in the normal range. All the patients were divided into 3 groups: the test group (24 GC patients and 24 healthy controls), the validation group (14 GC patients and 14 healthy control) and the additional group (87 GC patients). There were no significantly different in the basic clinicopathological factors, such as age, sex, BMI, etc between GC patients and healthy people in both the test and the validation group. Both the test and the validation group were investigated to compare the differences serum metabolites between GC patients and healthy controls in order to find the potential specific biomarkers for GC. Besides, blood samples of all the 125 patients were analyzed to find the relationship between biological metabolites and clinical parameters of GC and find specific prognostic factors for GC.

All the included GC patients had a complete 5-year follow-up record and did not have any hormone therapy or chemotherapy before, and all of them were with no significant acute inflammatory disease, normal liver and kidney function, routine physical status, normal results of biochemical tests and electrocardiograp (ECG). The patients should not have congenital disease for the last 2 weeks like burns, severe trauma, and septic shock, metabolic diseases like diabetes, severe heart and lung, liver or kidney disease, neurological and psychiatric diseases, blood diseases like leukemia and anemia, chronic inflammatory diseases, infectious diseases like HIV, hepatitis, and active tuberculosis, or any acute illnesses or stress reactions, etc. The lactation and pregnancy or possible pregnancy women, drinker, drug addicts, long-term user of proton pump inhibitors, hormones or 
non-steroidal anti-inflammatory agents should also be excluded. All the included healthy peoples should be of good health with no obvious abnormalities in routine physical examinations. The study protocol was authorized and all the procedures performed in this study involving human participants were in strict consistence with the ethical standards of ethics Committee at the xxx Hospital and with the 1964 Helsinki declaration and its later amendment. Well-written informed consent was obtained from all the participants prior to their enrollments.

\section{Sample procession and detection method}

A volume of 100ul serum samples were thawed, deproteinized with the volume of 400ul acetonitrile, and centrifuged at $14000 \mathrm{r} / \mathrm{min}$ for 5 minutes. Each sample was processed by Agilent 1200 high performance liquid chromatography combined with a 6520 accurate electrospray ionization /quadrupole-time-of-flight mass system (Agilent Technologies, California, USA). Serum samples were separated on an Eclipse Plus C18 column (2.1x150mm, $3.5 \mu \mathrm{m}$, Agilent Technologies, USA), with the condition of $180 \mu \mathrm{l}$ injection volume, $0.8 \mathrm{ml} / \mathrm{min}$ flow rate and $45^{\circ} \mathrm{C}$ column temperature, by using a gradient program of the mobile phase A $0.1 \%$ formic acid solution $\left(\mathrm{ESI}^{+}\right) /$water $\left(\mathrm{ESI}^{-}\right)$and mobile phase $\mathrm{B}$ was acetonitrile with $0.1 \%$ formic acid solution $\left(\mathrm{ESI}^{+}\right) /$acetonitrile (Merck, Darmstadt, Germany) (ESI $\left.{ }^{-}\right)$. The gradient program started from 20\% B for 0-1.5 min, linear increased from 20 to $95 \% \mathrm{~B}$ for $1.5-7 \mathrm{~min}$, stayed at $95 \% \mathrm{~B}$ for $7-9.9 \mathrm{~min}$, and then linear decreased from 95 to $20 \%$ B for 9.9-10 min and equilibrated for $20 \%$ B for 10-11 min. To avoid crosscontamination from GC patients, all the serum samples of healthy people were injected at the end.

All the data were collected in ionization quadrupoletime-of-flight mass spectrometry with both positive $\left(\mathrm{ESI}^{+}\right)$and negative $\left(\mathrm{ESI}^{-}\right)$full scan mode find either basic or acidic biological compounds in human serum, which may be specific and sensitive biomarkers for GC. The conditions of mass spectrometry were as following: the capillary voltage $3.2 \mathrm{kv}$; the cone voltage $35 \mathrm{~V}$; the desolvation temperature $350^{\circ} \mathrm{C}$; the source temperature was $100^{\circ} \mathrm{C}$; the desolvation gas (nitrogen) flow rate $650 \mathrm{~L} / \mathrm{h}$; the cone gas (nitrogen) flow rate was $50 \mathrm{~L} / \mathrm{h}$; a mass range of 50 to 1000 ; scan time of $1 \mathrm{~s}$ and inter-scan delay of $0.02 \mathrm{~s}$.

\section{Data processing and statistical analysis}

Firstly, both full-scan $\mathrm{ESI}^{+}$and $\mathrm{ESI}^{-}$raw mass spectra was gathered by using data-acquisition software Analyst TF 1.5.1 (AB Sciex, California, USA). Then, the data like retention time, peak area and $\mathrm{m} / \mathrm{z}$ ratio, was generated by using Marker View 1.2 (AB Sciex, California, USA) and the related serum metabolites were structurally confirmed by comparing the $\mathrm{m} / \mathrm{z}$ ratio and ion mode of those metabolites with data shown in HMDB (www.hmdb.ca) databases. Subsequently, principal component analysis (PCA) was adopted in score plots to make a distinction between the similarity or difference of the scatters between GC patients and healthy controls, and thereby two sample $t$-test by using SPSS 22.0 (SPSS Inc., Chicago, USA) was performed to select potential biological variables which statistically significant different between GC patients and healthy controls. Additionally, the hierarchical clustering analysis was implemented by using BRB-Array Tools (Dr. Richard Simo \& BRB-Array Tools Development Team, USA) to discriminate the subgroups and partial least squares discriminant analysis (PLS-DA) was applied by using simca-p software (Umetric AB, CA, USA) to identify the statistical important serum metabolites between GC patients and healthy controls. VIP plots of PLS-DA were drawn to ensure the correct potential serum biomarkers for both GC patients and healthy controls. The intensity of the background interference was normalized by using the global median subtraction method.

All the 125 GC patients were clustered into serum metabolites selected from the PLS-DA analysis, and differences in clinicopathological parameters like tumor differentiation, age, vascular invasion, TNM staging, survival rate, levels of Ki-67 and P53 and tumor position and expression were observed among the subgroups by chi-square test or variance analysis. The Kaplan-Meier (K-M) survival curve of each group was plotted based on different thresholds of sensitivity and specificity of survival time in order to find the most potential serum biomarkers for GC. Finally, multivariate COX regression analysis, variance analysis and K-M survival curve were used to find the independent prognostic factor for GC in human serum. All the statistical significance was set to be $p<0.05$.

\section{CONFLICTS OF INTEREST}

The authors declare no potential conflicts of interest.

\section{FUNDING}

This study was funded by the grant of National Natural Science Foundation of China (No. 81372295 \& No. 81402374).

\section{REFERENCES}

1. Ferro A, Peleteiro B, Malvezzi M, Bosetti C, Bertuccio P, Levi F, Negri E, La Vecchia C, Lunet N. Worldwide trends in gastric cancer mortality (1980-2011), with predictions to 2015, and incidence by subtype. Eur J Cancer. 2014; 50: 1330-1344. 
2. Jemal A, Bray F, Center MM, Ferlay J, Ward E, Forman D. Global cancer statistics. CA Cancer J Clin. 2011; 61: 69-90.

3. Jemal A, Siegel R, Ward E, Murray T, Xu J, Smigal C, Thun MJ. Cancer statistics, 2006. CA Cancer J Clin. 2006; 56: 106-130.

4. Cervantes A, Roda D, Tarazona N, Rosello S, Perez-Fidalgo JA. Current questions for the treatment of advanced gastric cancer. Cancer Treat Rev. 2013; 39: 60-67.

5. Noh SH, Park SR, Yang HK, Chung HC, Chung IJ, Kim SW, Kim HH, Choi JH, Kim HK, Yu W, Lee JI, Shin DB, Ji J, et al. Adjuvant capecitabine plus oxaliplatin for gastric cancer after D2 gastrectomy (CLASSIC): 5-year follow-up of an open-label, randomised phase 3 trial. Lancet Oncol. 2014; 15: 1389-1396.

6. Correa P. Gastric cancer: overview. Gastroenterol Clin North Am. 2013; 42: 211-217.

7. Thrumurthy SG, Chaudry MA, Hochhauser D, Mughal M. The diagnosis and management of gastric cancer. BMJ. 2013; 347: f6367.

8. Tashiro A, Sano M, Kinameri K, Fujita K, Takeuchi Y. Comparing mass screening techniques for gastric cancer in Japan. World J Gastroenterol. 2006; 12: 4873-4874.

9. Hu JD, Tang HQ, Zhang Q, Fan J, Hong J, Gu JZ, Chen JL. Prediction of gastric cancer metastasis through urinary metabolomic investigation using GC/MS. World J Gastroenterol. 2011; 17: 727-734.

10. Courant F, Antignac JP, Monteau F, Le Bizec B. Metabolomics as a potential new approach for investigating human reproductive disorders. J Proteome Res. 2013; 12: 2914-2920.

11. Manna SK, Krausz KW, Bonzo JA, Idle JR, Gonzalez FJ. Metabolomics reveals aging-associated attenuation of noninvasive radiation biomarkers in mice: potential role of polyamine catabolism and incoherent DNA damage-repair. J Proteome Res. 2013; 12: 2269-2281.

12. Wang X, Yang B, Sun H, Zhang A. Pattern recognition approaches and computational systems tools for ultra performance liquid chromatography-mass spectrometrybased comprehensive metabolomic profiling and pathways analysis of biological data sets. Anal Chem. 2012; 84: 428-439.

13. Zhang A, Sun H, Wang P, Han Y, Wang X. Modern analytical techniques in metabolomics analysis. Analyst. 2012; 137: 293-300.

14. Mondul AM, Moore SC, Weinstein SJ, Mannisto S, Sampson JN, Albanes D. 1-Stearoylglycerol is associated with risk of prostate cancer: results from serum metabolomic profiling. Metabolomics. 2014; 10: 1036-1041.

15. Jayavelu ND, Bar NS. Metabolomic studies of human gastric cancer: review. World J Gastroenterol. 2014; 20: 8092-8101.
16. Liang Q, Wang C, Li B. Metabolomic Analysis Using Liquid Chromatography/Mass Spectrometry for Gastric Cancer. App Biochem Biotechnol. 2015; 176: 2170-2184.

17. Chan AW, Gill RS, Schiller D, Sawyer MB. Potential role of metabolomics in diagnosis and surveillance of gastric cancer. World J Gastroenterol. 2014; 20: 12874-12882.

18. Wu H, Xue R, Tang Z, Deng C, Liu T, Zeng H, Sun Y, Shen X. Metabolomic investigation of gastric cancer tissue using gas chromatography/mass spectrometry. Anal Bioanal Chem. 2010; 396: 1385-1395.

19. Chen JL, Tang HQ, Hu JD, Fan J, Hong J, Gu JZ. Metabolomics of gastric cancer metastasis detected by gas chromatography and mass spectrometry. World J Gastroenterol. 2010; 16: 5874-5880.

20. Ikeda A, Nishiumi S, Shinohara M, Yoshie T, Hatano N, Okuno T, Bamba T, Fukusaki E, Takenawa T, Azuma T, Yoshida M. Serum metabolomics as a novel diagnostic approach for gastrointestinal cancer. BMC. 2012; 26: 548-558.

21. Song H, Peng JS, Dong-Sheng Y, Yang ZL, Liu HL, Zeng YK, Shi XP, Lu BY. Serum metabolic profiling of human gastric cancer based on gas chromatography/mass spectrometry. Braz J Med Biol Res. 2012; 45: 78-85.

22. Zhang Y, Gao G, Liu H, Fu H, Fan J, Wang K, Chen Y, Li B, Zhang C, Zhi X, He L, Cui D. Identification of volatile biomarkers of gastric cancer cells and ultrasensitive electrochemical detection based on sensing interface of Au-Ag alloy coated MWCNTs. Theranostics. 2014; 4: 154-162.

23. Antonowicz S, Kumar S, Wiggins T, Markar SR, Hanna GB. Diagnostic Metabolomic Blood Tests for Endoluminal Gastrointestinal Cancer--A Systematic Review and Assessment of Quality. Cancer Epidemiol Bio Prev. 2016; 25: 6-15.

24. Gu Y, Chen T, Fu S, Sun X, Wang L, Wang J, Lu Y, Ding S, Ruan G, Teng L, Wang M. Perioperative dynamics and significance of amino acid profiles in patients with cancer. J Transl Med. 2015; 13: 35-015-0408-1.

25. Asadi MH, Mowla SJ, Fathi F, Aleyasin A, Asadzadeh J, Atlasi Y. OCT4B1, a novel spliced variant of OCT4, is highly expressed in gastric cancer and acts as an antiapoptotic factor. Int J Cancer. 2011; 128: 2645-2652.

26. Vaananen $H$, Vauhkonen $M$, Helske $T$, Kaariainen I, Rasmussen M, Tunturi-Hihnala H, Koskenpato J, Sotka M, Turunen M, Sandstrom R, Ristikankare M, Jussila A, Sipponen P. Non-endoscopic diagnosis of atrophic gastritis with a blood test. Correlation between gastric histology and serum levels of gastrin-17 and pepsinogen I: a multicentre study. Eur J Gastroenterol Hepatol. 2003; 15: 885-891.

27. Kunisaki C, Shimada H, Nomura M, Matsuda G, Otsuka Y, Ono H, Akiyama H. Surgical outcome in patients with gastric adenocarcinoma in the upper third of the stomach. Surgery. 2005; 137: 165-171. 
28. Wang D, Ye F, Sun Y, Li W, Liu H, Jiang J, Zhang Y, Liu C, Tong W, Gao L, Sun Y, Zhang W, Seetoe T, et al. Protein signatures for classification and prognosis of gastric cancer a signaling pathway-based approach. Am J Pathol. 2011; 179: $1657-1666$.

29. Zhou Y, Li Y, Zheng J, Liu K, Zhang H. Detecting of gastric cancer by Bcl-2 and Ki67. Int J Clin Exp Pathol. 2015; 8: 7287-7290.
30. Miyagi Y, Higashiyama M, Gochi A, Akaike M, Ishikawa T, Miura T, Saruki N, Bando E, Kimura H, Imamura F, Moriyama M, Ikeda I, Chiba A, et al. Plasma free amino acid profiling of five types of cancer patients and its application for early detection. PLoS One. 2011; 6: e24143.

31. Pan C, Jia W, Lu B, Bishop CE. Expression of TAT recombinant Oct4, Sox2, Lin28, and Nanog proteins from baculovirusinfected Sf9 insect cells. Gene. 2015; 556: 245-248. 